\title{
SPORTS ANTHROPOLOGICAL AND SOMATOTYPICAL COMPARISON BETWEEN HIGHER CLASS MALE AND FEMALE BADMINTON AND TENNIS PLAYERS
}

\author{
Christoph RaschKa, Katharina Schmidt \\ Institute of Sports Sciences, Julius Maximilians University Würzburg, Germany
}

\begin{abstract}
The present study was to clarify whether there are sports anthropometric differences due to the different requirement profiles in tennis and badminton. 80 athletes ( 20 female and 20 male badminton players and 20 female and 20 male tennis players) were examined. Both badminton players and tennis players were from the second or third division. Anthropometric data and computed constitutional and somatotypical parameters in this work correspond to international standards.

The male badminton (tennis) players are characterized by the triplet combination $3.4-3.3-3.4(3.4-3.2-3.8)$ in the somatochart of Parnell, by the triplet combination $2.3-3.2-2.7(2.3-3.2-3.1)$ in the Heath \& Carter somatochart.

In the present study, the women's badminton average somatotype resulted by Parnell (Heath \& Carter) of $4.1-3.3-2.8$ (3.7 - 3.1 - 2.0), with the tennis ladies of $4.2-2.8-3.5(3.5-2.6-2.9)$.

In summary it can be said that the male and female badminton players compared with the tennis players were smaller and had shorter arms and shorter legs (but longer lower legs) and a longer torso. In addition, the body of badminton players appeared strong and robust, and the calf muscles seemed more pronounced.

Tennis players possessed significantly thicker and larger humeri and feet. In the somatotypology the picture is that of the tall, ectomorphic tennis player is compared to the smaller, but more robust badminton player.
\end{abstract}

Keywords: sports anthropological investigation, somatotypical investigation, badminton and tennis players 


\section{INTRODUCTION}

It is often claimed that badminton is the fastest racquet sport. Although badminton holds the record for the fastest initial speed of a racket sports projectile, the shuttlecock decelerates substantially faster than other projectiles such as tennis balls. While players of badminton or tennis often claim that their sport is the more physically demanding, such comparisons are difficult to make objectively because of the differing demands of the games. No formal study currently exists evaluating the physical condition of the players, their demands during game play and the sports anthropological differences.

The present study was to clarify whether due to the similarities between the two sports there is also a similar type of constitution in tennis players and badminton players, or whether there are sports anthropometric differences due to the different requirement profiles.
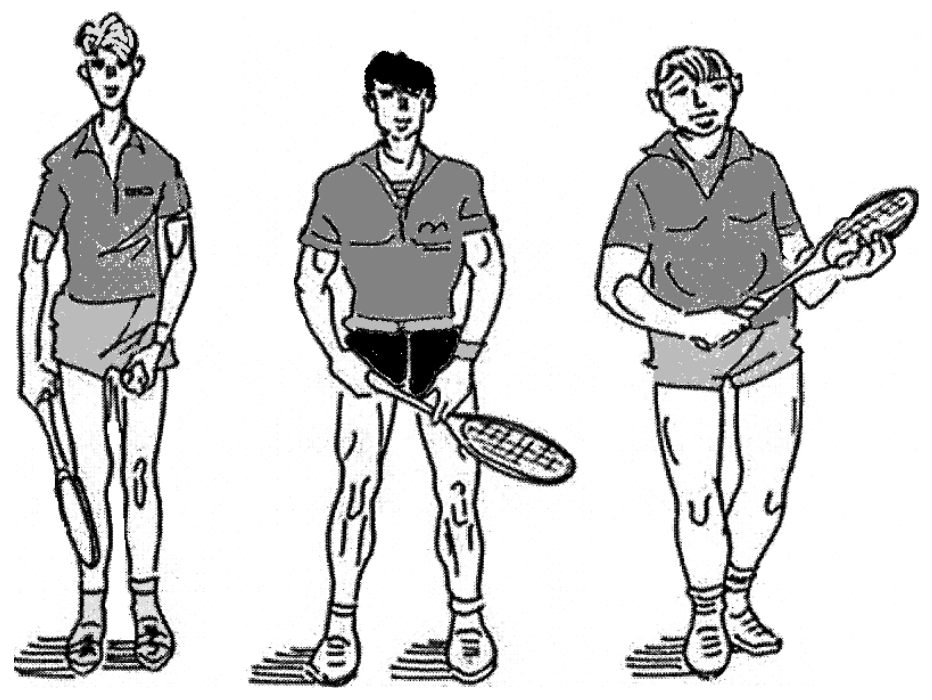

Figure 1.

Leptosom, Athletic and Pyknic Tennis Players (according to Pöttinger and Mensing 1986)

\section{PARTICIPANTS AND METHODS}

In this study 80 athletes (20 female and 20 male badminton players and 20 female and 20 male tennis players) were examined. Both badminton players and tennis players were from the second or third division. Each proband participated voluntarily and the data were used anonymously. Anthropometric data and computed constitutional and somatotypical parameters in this work correspond to international standards (Conrad 1963, Heath \& Carter 1967+1990, Knussmann 1996, Martin\&Knussmann 1988, Raschka 2006, 
Tittel\&Wutscherk 1972). The analysis of differences was tested by Anova, correlations were tested by the Pearson correlation coefficients.

\section{RESULTS}

The distribution of constitutional types after Conrad and Tittel \& Wutscherk (1972) and the somatotypes after Parnell and Heath \& Carter are summarized in Figures 2-4.
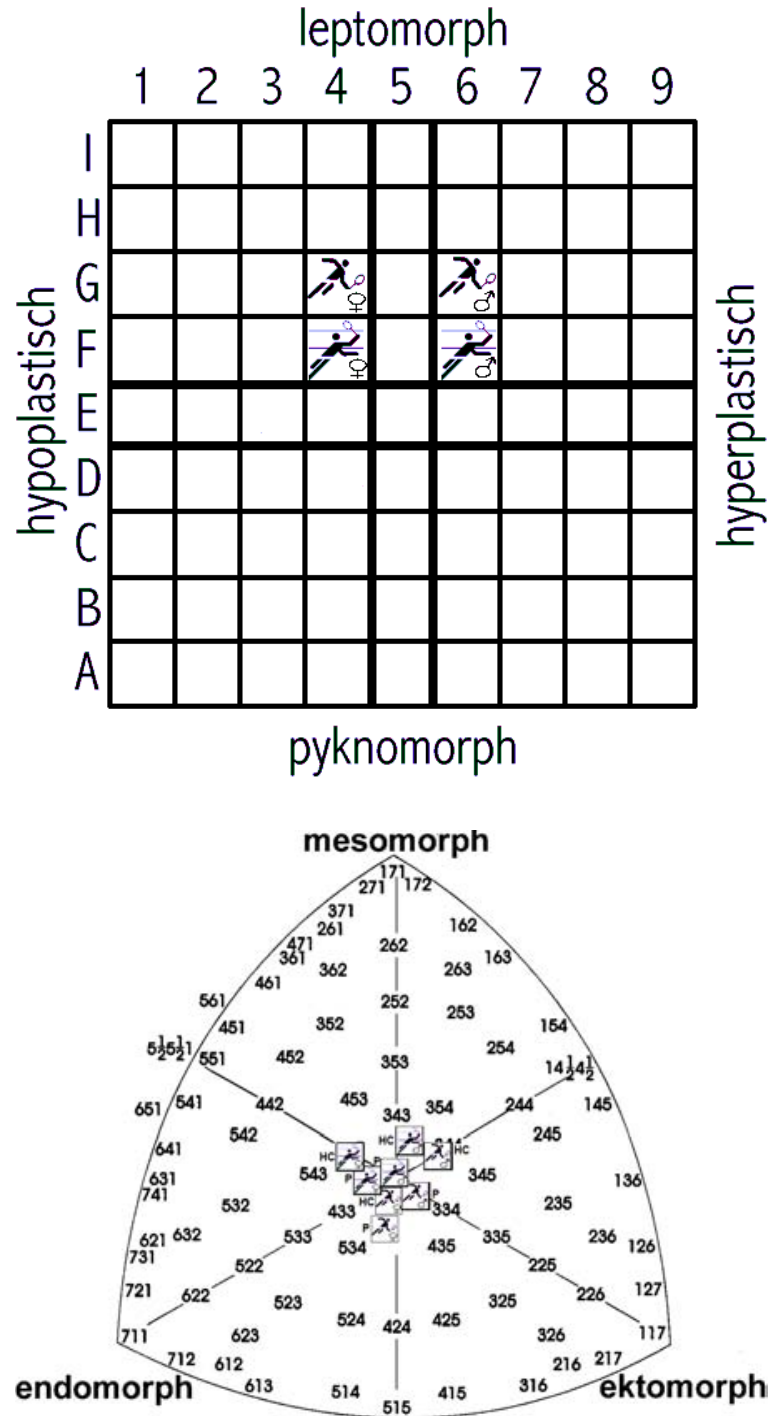

Figure 2: Male and female average constitutional types of tennis and badminton players in the chessboard pattern graphic after Conrad

Figure 3. Male and female average somatotypes of badminton and tennis players after Heath \& Carter ( $\mathrm{HC})$ and Parnell $(P)$ in the somatochart. 


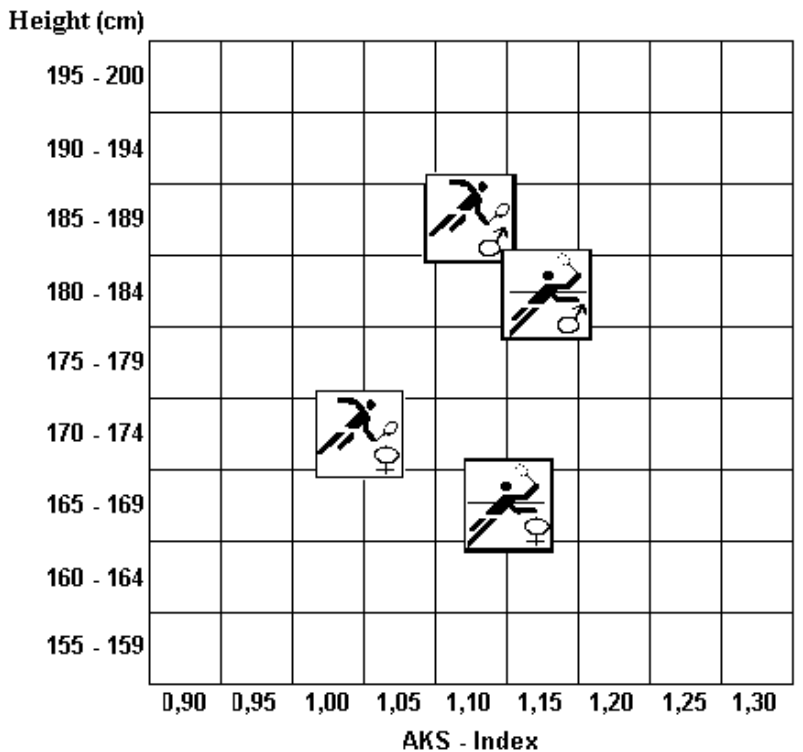

Figure 4. Male and female tennis and badminton players in the diagram after Tittel \& Wutscherk (1972).
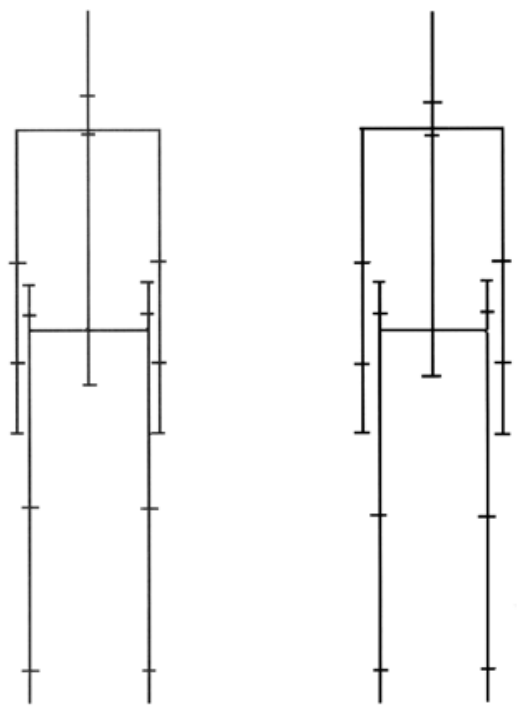

Figure 5. Proportion figures of female badminton (left) and tennis players (right).
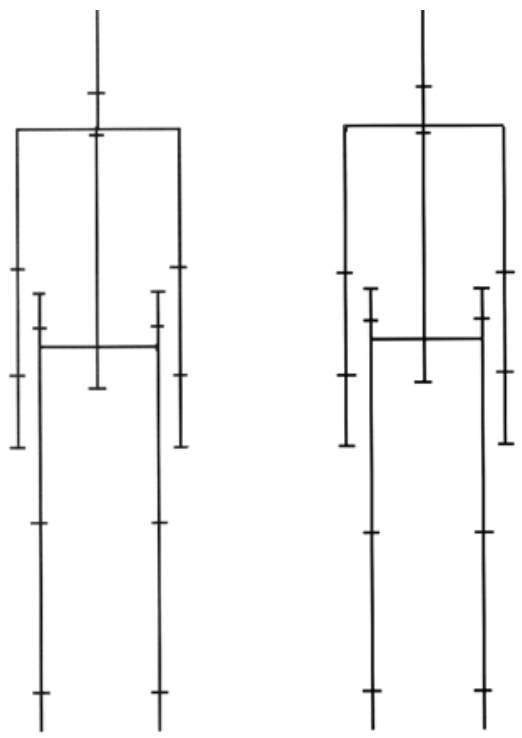

Figure 6. Proportion figures of male badminton (left) and tennis players (right). 
Table 1. Sports anthropometric parameters of male and female badminton and tennis players

\begin{tabular}{|c|c|c|c|c|c|}
\hline \multirow[b]{2}{*}{ Parameter } & \multicolumn{2}{|c|}{ Badminton } & \multicolumn{2}{|c|}{ Tennis } & \multirow[t]{2}{*}{$\mathbf{p}$} \\
\hline & Men & Women & Men & Women & \\
\hline Age (years) & $22.7 \pm 3.8$ & $24.0 \pm 2.4$ & $22.8 \pm 3.3$ & $21.1 \pm 4-3$ & n.s. \\
\hline Height (Vertex; cm) & $182.0 \pm 4.6$ & $168.1 \pm 5.8$ & $184.8 \pm 4.8$ & $170.1 \pm 4.2$ & $<0.05$ \\
\hline Gnathion $(\mathrm{cm})$ & $160.3 \pm 4.5$ & $147.5 \pm 4.9$ & $163.3 \pm 4.3$ & $148.1 \pm 4.0$ & n.s. \\
\hline Suprasternale (cm) & $148.5 \pm 4.0$ & $137.1 \pm 5.3$ & $150.5 \pm 4.2$ & $137.8 \pm 3.5$ & n.s. \\
\hline Acromiale $(\mathrm{cm})$ & $151.3 \pm 3.6$ & $139.0 \pm 5.3$ & $153.9 \pm 4.7$ & $141.3 \pm 3.9$ & $<0.05$ \\
\hline Radiale (cm) & $117.0 \pm 3.1$ & $107.3 \pm 4.3$ & $118.0 \pm 4.5$ & $109.2 \pm 3.8$ & n.s. \\
\hline Stylion $(\mathrm{cm})$ & $90.1 \pm 2.7$ & $83.8 \pm 3.7$ & $91.7 \pm 4.0$ & $84.7 \pm 3.3$ & n.s. \\
\hline Dactylion $(\mathrm{cm})$ & $71.6 \pm 2.6$ & $66.8 \pm 3.1$ & $72.8 \pm 3.6$ & $67.9 \pm 3.1$ & n.s. \\
\hline Iliocristale (cm) & $110.0 \pm 3.4$ & $101.5 \pm 4.4$ & $111.6 \pm 4.2$ & $102.2 \pm 3.3$ & n.s. \\
\hline Iliospinale $(\mathrm{cm})$ & $101.8 \pm 3.5$ & $93.8 \pm 4.3$ & $104.1 \pm 4.2$ & $95.4 \pm 3.0$ & $<0.05$ \\
\hline Trochanterion $(\mathrm{cm})$ & $92.6 \pm 3.5$ & $85.0 \pm 4.2$ & $94.8 \pm 4.7$ & $87.6 \pm 2.9$ & $<0.01$ \\
\hline Tibiale $(\mathrm{cm})$ & $51.9 \pm 2.2$ & $47.1 \pm 2.4$ & $50.6 \pm 1.9$ & $45.9 \pm 2.6$ & $<0.05$ \\
\hline Sphyrion $(\mathrm{cm})$ & $9.1 \pm 0.9$ & $8.2 \pm 0.7$ & $9.3 \pm 0.8$ & $8.3 \pm 0.7$ & n.s. \\
\hline Sitting height $(\mathrm{cm})$ & $95.9 \pm 2.5$ & $89.9 \pm 3.3$ & $95.7 \pm 3.2$ & $89.8 \pm 2.5$ & n.s. \\
\hline Arm span $(\mathrm{cm})$ & $184.8 \pm 5.8$ & $168.0 \pm 6.2$ & $188.1 \pm 6.0$ & $171.0 \pm 5.3$ & $<0.05$ \\
\hline Shoulder width $(\mathrm{cm})$ & $39.2 \pm 1.4$ & $33.7 \pm 1.5$ & $38.8 \pm 1.7$ & $33.9 \pm 1.6$ & n.s. \\
\hline Chest width $(\mathrm{cm})$ & $31.0 \pm 1.6$ & $28.0 \pm 1.7$ & $30.3 \pm 1.8$ & $27.3 \pm 1.5$ & $<0.05$ \\
\hline Chest depth $(\mathrm{cm})$ & $20.8 \pm 2.3$ & $17.8 \pm 1.0$ & $19.7 \pm 1.6$ & $17.3 \pm 1.7$ & $<0.05$ \\
\hline Pelvis width $(\mathrm{cm})$ & $28.0 \pm 1.2$ & $27.8 \pm 1.5$ & $27.8 \pm 1.4$ & $26.6 \pm 1.1$ & $<0.05$ \\
\hline Epiphysis width Femur (cm) & $8.9 \pm 0.4$ & $8.4 \pm 0.4$ & $9.0 \pm 0.6$ & $8.3 \pm 0.5$ & n.s. \\
\hline Ancle breadth & $7.7 \pm 0.4$ & $6.8 \pm 0.3$ & $7.6 \pm 0.4$ & $6.6 \pm 0.5$ & n.s. \\
\hline Epiphysis width Humerus (cm) & $6.3 \pm 0.4$ & $5.6 \pm 0.3$ & $6.7 \pm 0.4$ & $5.8 \pm 0.3$ & $<0.001$ \\
\hline Hand breadth $(\mathrm{cm})$ & $7.9 \pm 0.4$ & $7.1 \pm 0.4$ & $8.1 \pm 0.4$ & $7.2 \pm 0.4$ & n.s. \\
\hline Neck circumference $(\mathrm{cm})$ & $38.1 \pm 1.8$ & $33.4 \pm 1.6$ & $38.1 \pm 1.6$ & $32.6 \pm 1.5$ & n.s. \\
\hline $\begin{array}{l}\text { Chest circumference } \\
\text { (respiratory centre, } \mathrm{cm} \text { ) }\end{array}$ & $96.1 \pm 4.3$ & $87.1 \pm 4.3$ & $95.5 \pm 5.2$ & $85.4 \pm 3.4$ & n.s. \\
\hline $\begin{array}{l}\text { Chest circumference in } \\
\text { inspiration }(\mathrm{cm})\end{array}$ & $101.7 \pm 4.6$ & $93.1 \pm 4.1$ & $101.2 \pm 4.7$ & $90.7 \pm 3.8$ & n.s. \\
\hline $\begin{array}{l}\text { Chest circumference in } \\
\text { exspiration }(\mathrm{cm})\end{array}$ & $92.7 \pm 4.3$ & $84.7 \pm 3.9$ & $92.2 \pm 5.2$ & $83.5 \pm 3.6$ & n.s. \\
\hline Waist circumference $(\mathrm{cm})$ & $81.6 \pm 3.8$ & $73.5 \pm 3.6$ & $79.8 \pm 4.0$ & $69.6 \pm 3.7$ & $<0.001$ \\
\hline Pelvis circumference $(\mathrm{cm})$ & $90.1 \pm 4.1$ & $87.4 \pm 4.6$ & $90.6 \pm 4.6$ & $85.1 \pm 3.2$ & n.s. \\
\hline $\begin{array}{l}\text { Upper arm circumference in } \\
\text { flexion }(\mathrm{cm}) \text {, left side }\end{array}$ & $31.8 \pm 2.1$ & $27.6 \pm 1.9$ & $31.9 \pm 3.0$ & $27.2 \pm 1.8$ & n.s. \\
\hline $\begin{array}{l}\text { Upper arm circumf. flex. } \\
\text { (cm),non-dominant side }\end{array}$ & $31.6 \pm 1.9$ & $27.6 \pm 1.9$ & $31.9 \pm 3.0$ & $27.2 \pm 1.8$ & n.s. \\
\hline
\end{tabular}




\begin{tabular}{|c|c|c|c|c|c|}
\hline \multirow[b]{2}{*}{$\begin{array}{l}\text { Upper arm circumf. in flexion } \\
\text { (cm), right side }\end{array}$} & \multicolumn{2}{|c|}{ Badminton } & \multicolumn{2}{|c|}{ Tennis } & \multirow{2}{*}{$\frac{\mathbf{p}}{\text { n.s. }}$} \\
\hline & $32.8 \pm 1.9$ & $28.9 \pm 1.9$ & $33.0 \pm 3.0$ & $28.2 \pm 1.9$ & \\
\hline $\begin{array}{l}\text { Upper arm circumf. flex. }(\mathrm{cm}) \text {, } \\
\text { dominant side }\end{array}$ & $32.9 \pm 1.9$ & $28.9 \pm 1.9$ & $33.0 \pm 3.0$ & $28.2 \pm 1.9$ & n.s. \\
\hline $\begin{array}{l}\text { Upper arm circumference } \\
\text { extension }(\mathrm{cm}) \text {, left side }\end{array}$ & $28.4 \pm 2.1$ & $26.3 \pm 1.7$ & $29.0 \pm 2.7$ & $25.4 \pm 1.9$ & n.s. \\
\hline $\begin{array}{l}\text { Upper arm circumference } \\
\text { extension }(\mathrm{cm}) \text {,right side }\end{array}$ & $29.2 \pm 1.8$ & $26.8 \pm 1.8$ & $29.8 \pm 2.7$ & $25.9 \pm 2.0$ & n.s. \\
\hline $\begin{array}{l}\text { Forearm circumference } \\
\text { maximum }(\mathrm{cm}), \text { left side }\end{array}$ & $27.0 \pm 1.4$ & $23.6 \pm 1.1$ & $27.0 \pm 1.8$ & $23.7 \pm 1.2$ & n.s. \\
\hline $\begin{array}{l}\text { Forearm circumf. max. }(\mathrm{cm}) \text {, } \\
\text { non-dominant side }\end{array}$ & $26.8 \pm 1.2$ & $23.6 \pm 1.1$ & $26.9 \pm 1.8$ & $23.7 \pm 1.2$ & n.s. \\
\hline $\begin{array}{l}\text { Forearm circumference } \\
\text { maximum }(\mathrm{cm}) \text { right side }\end{array}$ & $28.1 \pm 1.2$ & $25.2 \pm 1.2$ & $28.6 \pm 1.8$ & $25.0 \pm 1.4$ & n.s. \\
\hline $\begin{array}{l}\text { Forearm circumf. maximum } \\
(\mathrm{cm}) \text {, dominant side }\end{array}$ & $28.2 \pm 1.2$ & $25.2 \pm 1.2$ & $28.6 \pm 1.7$ & $25.1 \pm 1.3$ & n.s. \\
\hline $\begin{array}{l}\text { Forearm circumference } \\
\text { minimum }(\mathrm{cm})\end{array}$ & $16.9 \pm 0.7$ & $15.2 \pm 0.5$ & $17.0 \pm 0.6$ & $15.2 \pm 0.6$ & n.s. \\
\hline Hand circumference $(\mathrm{cm})$ & $20.9 \pm 0.9$ & $18.7 \pm 0.8$ & $21.4 \pm 1.2$ & $18.7 \pm 0.8$ & n.s. \\
\hline Thigh circumference $(\mathrm{cm})$ & $52.3 \pm 3.0$ & $52.1 \pm 2.7$ & $52.4 \pm 3.0$ & $51.1 \pm 2.9$ & n.s. \\
\hline Calf circumference $(\mathrm{cm})$ & $37.9 \pm 1.6$ & $37.4 \pm 1.6$ & $37.4 \pm 2.0$ & $35.9 \pm 3.1$ & $<0.05$ \\
\hline $\begin{array}{l}\text { Lower leg circumference } \\
\text { minimum }(\mathrm{cm})\end{array}$ & $23.8 \pm 1.1$ & $23.0 \pm 1.1$ & $24.9 \pm 1.4$ & $22.3 \pm 1.1$ & n.s. \\
\hline $\begin{array}{l}\text { Morphological facial height } \\
(\mathrm{cm})\end{array}$ & $11.6 \pm 0.8$ & $10.8 \pm 0.6$ & $11.8 \pm 0.7$ & $11.0 \pm 0.5$ & n.s. \\
\hline Zygomatic breadth (cm) & $12.3 \pm 0.6$ & $11.9 \pm 0.5$ & $12.6 \pm 0.4$ & $11.8 \pm 0.5$ & n.s. \\
\hline Foot length $(\mathrm{cm})$ & $26.0 \pm 0.9$ & $24.0 \pm 1.0$ & $26.9 \pm 0.8$ & $24.2 \pm 0.7$ & $<0.01$ \\
\hline Foot width $(\mathrm{cm})$ & $10.7 \pm 0.5$ & $9.7 \pm 0.7$ & $10.5 \pm 0.7$ & $10.0 \pm 0.8$ & n.s. \\
\hline Subscapular skinfold (mm) & $9.4 \pm 2.0$ & $11.1 \pm 3.0$ & $9.7 \pm 1.9$ & $11.3 \pm 2.9$ & n.s. \\
\hline Triceps skinfold $(\mathrm{mm})$ & $7.4 \pm 2.0$ & $11.4 \pm 2.2$ & $7.3 \pm 2.8$ & $12.0 \pm 3.2$ & n.s. \\
\hline Forearm skinfold $(\mathrm{mm})$ & $4.2 \pm 0.6$ & $4.2 \pm 0.6$ & $4.1 \pm 0.4$ & $4.2 \pm 0.7$ & n.s. \\
\hline Suprailiac skinfold (mm) & $8.1 \pm 2.2$ & $13.2 \pm 4.7$ & $8.4 \pm 2.7$ & $11.0 \pm 3.2$ & n.s. \\
\hline Thigh skinfold (mm) & $7.1 \pm 2.2$ & $12.3 \pm 3.3$ & $6.8 \pm 2.0$ & $11.4 \pm 3.1$ & n.s. \\
\hline Calf skinfold (mm) & $7.9 \pm 2.3$ & $14.2 \pm 3.4$ & $7.8 \pm 2.7$ & $13.7 \pm 3.4$ & n.s. \\
\hline $\begin{array}{l}\text { Body fat percentage } \\
\text { (calipermetry; \%) }\end{array}$ & $10.8 \pm 1.9$ & $18.9 \pm 2.1$ & $11.1 \pm 2.2$ & $18.3 \pm 2.0$ & n.s. \\
\hline Body fat percentage (BIA; \%) & $21.7 \pm 4.0$ & $33.0 \pm 4.0$ & $20.6 \pm 4.5$ & $28.7 \pm 4.8$ & $<0.01$ \\
\hline Plastik-Index after Conrad & $86.9 \pm 3.0$ & $76.0 \pm 3.0$ & $87.1 \pm 3.9$ & $76.2 \pm 2.9$ & n.s. \\
\hline Metrik-Index after Conrad & $-0.6 \pm 0.4$ & $-0.7 \pm 0.3$ & $-1.0 \pm 0.3$ & $-1.0 \pm 0.4$ & $<0.001$ \\
\hline Pyknomorphy after Knußmann & $-2.2 \pm 1.7$ & $-1.0 \pm 1.2$ & $-2.7 \pm 1.0$ & $-1.6 \pm 1.2$ & $<0.05$ \\
\hline Makrosomia after Knußmann & $3.8 \pm 1.3$ & $3.9 \pm 1.4$ & $4.3 \pm 1.6$ & $4.2 \pm 1.4$ & n.s. \\
\hline Endomorphy after Parnell & $3.4 \pm 0.6$ & $4.1 \pm 0.8$ & $3.4 \pm 0.7$ & $4.2 \pm 0.6$ & n.s. \\
\hline Mesomorphy after Parnell & $3.3 \pm 1.0$ & $3.3 \pm 0.7$ & $3.2 \pm 1.0$ & $2.8 \pm 1.2$ & n.s. \\
\hline
\end{tabular}




\begin{tabular}{lcc|cc|c}
\hline & \multicolumn{2}{c}{ Badminton } & \multicolumn{2}{c}{ Tennis } & p \\
\hline Ectomorphy after Parnell & $3.4 \pm 0.8$ & $2.8 \pm 0.9$ & $3.8 \pm 0.8$ & $3.5 \pm 1.0$ & $<0.01$ \\
\hline $\begin{array}{l}\text { Endomorphy after } \\
\text { Heath\&Carter }\end{array}$ & $2.3 \pm 0.6$ & $3.7 \pm 0.8$ & $2.3 \pm 0.6$ & $3.5 \pm 0.7$ & n.s. \\
\hline $\begin{array}{l}\text { Mesomorphy after } \\
\text { Heath\&Carter }\end{array}$ & $3.2 \pm 0.9$ & $3.1 \pm 0.8$ & $3.2 \pm 0.9$ & $2.6 \pm 1.1$ & n.s. \\
\hline $\begin{array}{l}\text { Ectomorphy after } \\
\text { Heath\&Carter }\end{array}$ & $2.7 \pm 0.8$ & $2.0 \pm 0.8$ & $3.1 \pm 0.8$ & $2.9 \pm 1.0$ & $<0.001$ \\
\hline Body weight $(\mathrm{kg})$ & $77.5 \pm 5.9$ & $65.5 \pm 6.6$ & $78.1 \pm 8.5$ & $62.1 \pm 6.3$ & n.s. \\
\hline BMI $\left(\mathrm{kg} / \mathrm{m}^{2}\right)$ & $23.4 \pm 1.6$ & $23.2 \pm 1.9$ & $22.8 \pm 1.8$ & $21.5 \pm 1.9$ & $<0.01$ \\
\hline Pelidisi-Index $(\mathrm{kg} / \mathrm{cm})$ & $95.7 \pm 2.9$ & $96.6 \pm 2.9$ & $96.2 \pm 3.5$ & $95.0 \pm 3.6$ & n.s. \\
\hline
\end{tabular}

\section{DISCUSSION}

Unlike Klingler and Biener (1986) who examined players from lower leagues, in the present study, a larger body height of male and female tennis players was found compared to badminton. WEBER described in 1987 with his measurements for tennis players $(183.3 \mathrm{~cm})$ and badminton players $(181.4 \mathrm{~cm})$ similar results.

The importance of the body height in tennis is reflected in the results of Copley (1980): The pros were with an average size of $182.8 \mathrm{~cm}$ much larger than the amateurs with $178.5 \mathrm{~cm}$. In the study of Pallulat (1984) top tennis players were on average $170.5 \mathrm{~cm}$ tall.

After Stockhausen (1999) the body length in women's tennis seems to determine more clearly the performance than in men's tennis.

The body heights in badminton (men and women) are essentially in agreement with the results for the Czech national team, which was investigated by Heller and Koudelkova (2003).

Concerning the measurements of the widths in the present study, we found a significantly lower arm span of badminton players compared to the tennis players.

The larger calf circumferences of the badminton players are a sign of more pronounced calf muscles.

Also, the foot of the tennis players was significantly longer than for the badminton players.

Calipermetrically there was no significant difference between the sports.

The male badminton (tennis) players are characterized by the triplet combination $3.4-3.3-3.4(3.4-3.2-3.8)$ in the somatochart of Parnell, by the 
triplet combination $2.3-3.2-2.7(2.3-3.2-3.1)$ in the Heath \& Carter somatochart.

Withers et al. found in 1986 at South Australian male (female) badminton players a mean somatotype of $2.5-4.6-3.2(4.1-4.4-2.5)$.

COPLEY describes in 1980 for the tennis players of the South Africa Open the somatotypes of $2.2-4.6-3.0$ for the professionals and $2.2-4.3-3.2$ for the amateurs.

In the present study, the women's badminton average somatotype resulted by Parnell (Heath \& Carter) of $4.1-3.3-2.8(3.7-3.1-2.0)$, with the tennis ladies of $4.2-2.8-3.5(3.5-2.6-2.9)$. Copley (1980) described in South African women's tennis average somatotypes of $3.1-3.9-2.6$ for professionals and $2.6-3.2-3.6$ for amateurs.

In summary it can be said that the male and the female badminton players compared with the tennis players were smaller and had shorter arms and shorter legs (but longer lower legs) and a longer torso. In addition, the body of badminton players appeared strong and robust, and the calf muscles seemed more pronounced.

Tennis players possessed significantly thicker and larger humeri and feet. In the somatotypology the picture is that of the tall, ectomorphic tennis player compared to the smaller, but more robust badminton player.

\section{REFERENCES}

1. Conrad K. (1963). Der Konstitutionstypus. Berlin. Springer Verlag

2. Copley B. B. (1980). An anthropometric, somatotypical and physiological study of tennis players with special reference to the effects of training. $\mathrm{PhD}$ Thesis. University of the Witwaterstrand, Johannesburg.

3. Copley B. B. (1980). A morphological and physiological study of tennis players with special reference to the effects of training. South African Journal for Research in Sports, Physical Education and Recreation 3, 33-44.

4. Heath B. H., Carter L. J. E. (1967). A modified somatotype method. Am J Phys Anthrop 27, 57-74.

5. Heath B. H., Carter L. J. E. (1990). Somatotyping-development and applications. Cambridge Studies in Biological Anthropology. Great Britain. Redwood Press.

6. Heller J., Koudelkova M. (2003). Physiological profiles of elite male and female badminton players. Kinanthropologica 39, 63-75.

7. Klingler K., Biener K. (1986). Zur Sportmedizin des Badminton. Österreichisches Journal für Sportmedizin 16, 24-32. 
8. Knußmann R. (1996). Vergleichende Biologie des Menschen. Lehrbuch der Anthropologie. Stuttgart. Gustav Fischer Verlag.

9. Kretschmer E. (1921). Körperbau und Charakter. Berlin. Springer Verlag.

10. Martin R. \& Knussmann R. (1988). Anthropologie. Handbuch. Band I. Stuttgart. Fischer Verlag.

11. Pallulat D. M. A. (1984). Physiques of female professional tennis players. MA Thesis. San Diego State University.

12. Parnell R. W. (1954) Somatotyping by physical anthropometry. Am J Phys Anthrop 12, 209-239.

13. Pöttinger P., Mensing E. (1986). Tennissport. Handbuch für Lehre, Training und Spiel. Böblingen: Central-Druck Verlagsgesellschaft.

14. Raschka C. (2006). Sportanthropologie. Köln. Sportverlag Strauß.

15. Stockhausen W. (1999). Besondere sportmedizinische Aspekte im Nachwuchsbereich. In: Hölting N., Mester J. (Eds.). Belastung und Regeneration im Tennis. 7. Symposium des Ausschusses für Sportwissenschaft des DTB vom 13.-14.11.1998 in Göttingen. p 39-51. Hamburg. Czwalina Verlag.

16. Tittel K., Wutscherk H. (1972). Sportanthropometrie. Leipzig. Barth.

17. Weber K. (1987). Der Tennissport aus internistisch-sportmedizinischer Sicht. Band 20: Schriften der Deutschen Sporthochschule Köln. Sankt Augustin. Hans Richarz Verlag.

18. Withers R. T., Whittingham N. O., Norton K. I. (1987). Relative Body Fat and anthropometric prediction of body density of female athletes. European Journal of Applied Physiology 56 (2), 169-180.

19. Withers R. T., Craig N. P., Bourdon P. C. (1987). Relative Body Fat and anthropometric prediction of body density of male athletes. European Journal of Applied Physiology 56 (2), 191-200.

\section{Adress for correspondence}

Christoph Raschka

Institute of Sports Sciences

Julius-Maximilians-University

Judenbühlwegn 11 D-97082 Würzburg, Germany

E-mail: christoph.raschka@uni-wuerzburg.de 